\title{
Predictors of Health Related Quality of Life for Adults Ages 40-64
}

\section{Stefany H Almaden*}

Health Services Consultant/President, The Almaden Group, Inc., Care Management \& Transitional Leadership Consultants, P.O. Box 5095, Pasadena, CA 91117, USA

\begin{abstract}
Background: This study focused on access to care for adults 40 to 64 with the purpose of identifying predictors of health-related quality of life (HR-QoL). The literature had inconsistencies and gaps in experimental methoods in determining HR-QoL predictors.
\end{abstract}

Methods: Using Becker's HBM as its theoretical framework as well as Quality Metric instrument SF 12 vs $2^{\text {TM }}$ for measuring HR-QoL, this explorative correlational study examined under insurance, continued health coverage, and health behavior in relation to HR-QoL while controlling for personal attributes like age, gender, race/ethnicity, and level of income and education. Multivariate regression analyses were conducted with a sample $\mathrm{N}=165$.

Results: The study focused on three research question with three Null and Alternate hypotheses in search of predictors of HR-QoL. Although no significant relations were observed when linking the three predictors to HR-QoL, significant positive association between health seeking behavior and continuity of health coverage was observed.

Conclusion: Continuity of health coverage had a significant relation to health seeking behavior with implications for positive social change. An understanding of factors that contribute to health behavior and engaging patients to seek health services are key factors to improving health status.

Keywords: Access; Health behavior; Continuity of care; Underinsurance; High deductible health plans; HR-QoL

\section{Background}

The Institute of Medicine [1] called for timely access through preventive care, health screening and early diagnostics as a way of yielding better health outcomes. Currently, pay for performance (P4P) strategies to improve health outcomes while controlling costs of managing chronic conditions through a focus on timely access to care place added emphasis on this strategy [2]. In this study, I sought to explore this access to care further.

The challenge faced by the health care community was defining factors that influence health status in a way that allows effective interventions. The health services literature referenced multiple factors influencing health. These included timely access to care, personal attributes, health seeking behavior, affordability of care, type of health plan insurance coverage with particular focus on the uninsured and underinsured, and cost of services that drive decisions to seek care $[3,4]$. In this study, I explored predictors of health factors and the strength of their relationship in relation to health status.

\section{Problem Statement}

The study was to address access to care for adults 40 to 64 with the purpose of identifying predictors of health-related quality of life (HR-QoL). Delayed access to diagnostic or therapeutic care yields an increased financial burden for treating illness [5]. Furthermore, delayed access to care and its relationship to HR-QoL were particular concerns among people age 40 to 64 that were also classified as the working poor or underinsured [6].

The 40-64 age group faced challenges in having continued health insurance coverage as a result of job loss, career transitions, or limited resources [6]. Much of the research focused either on the recipients of publicly funded health plans such as Medicare, Medicaid, or on the uninsured. Nonetheless, health coverage remains a challenge.

In addition to exploring the relationship between underinsurance and HR-QoL, I examined the relationship between continuity of health coverage and HR-QoL. While continuity of care has been associated with better health outcomes, there was a gap in the literature when it came to empirically measuring that relationship. The literature addressed personal characteristics as variables that impact health status but there was no consistency in findings.

Therefore, the gap to fill with this study was an empirical assessment of the relationship of underinsurance status, continuity of health coverage, and health-seeking behavior to HR-QoL. Also, another gap to fill was testing the commonly used health behavior tool for validity and reliability. This combined empirical approach focused on all variables that correlate with HR-QoL had not previously been undertaken which was a main purpose of this study. Furthermore, the study had a purpose to help policy makers, clinicians, employers, and individuals make informed choices about health coverage and health services delivery that provides cost control, quality of care, and better health outcomes through an understanding of predictors of HR-QoL.

\section{Relevant Literature}

In order to understand the meaning of health, I researched multiple databases such as Health Services, Public Policy, Social-Index, and Psychology using keywords that included affordability of care, access to care, health, underinsured, health beliefs, personal attributes, and healthrelated quality of life. I also researched websites that served as data mining sources for studies examining factors that impact health.

The literature focused on health plan type such as high-deductible health plans (HDHP) and health behaviors like avoidance of seeking care due cost $[1,3,4]$. The literature also focused on personal characteristics and adherence to treatment plans for specific populations like diabetics or cardiac [7-10]. However, findings were inconsistent.

*Corresponding author: Dr. Stefany $\mathrm{H}$. Almaden, PhD, RN, MSN, CCM, CPUM, CMCN, Health Services Consultant/President, The Almaden Group Inc., Care Management \& Transitional Leadership Consultants, P.O. Box 5095, Pasadena, CA 91117, USA, Tel: 626-388-7469; Fax: 626-791-0920; E-mail: stefany@almadencare.com

Received July 05, 2013; Accepted August 30, 2013; Published September 09 2013

Citation: Almaden SH (2013) Predictors of Health Related Quality of Life for Adults Ages 40-64. J Nurs Care 2: 133. doi:10.4172/2167-1168.1000133

Copyright: (c) 2013 Almaden SH. This is an open-access article distributed under the terms of the Creative Commons Attribution License, which permits unrestricted use, distribution, and reproduction in any medium, provided the original author and source are credited. 
Spiraling health care costs and the effect on individuals' decisions to avoid or delay seeking health services resulted in declining healthrelated quality of life (HR-QoL) $[11,12]$. Costs further led to disruptions in continued health coverage which also reduced HR-QoL $[12,13]$. Reduced HR-QoL, according to Hoffman et al. [12], was prevalent among individuals age 40-64 that were referred to as the working poor.

These individuals often face challenges in continuity of health coverage due to job loss, career transitions, or declining income $[1,11]$.

\section{Health care status}

As health care spending mounts, the complexity of access to care continues. The World Health Organization (WHO) called for the "new and different demands" on the health delivery system resulting from chronicity of illness and necessary clinicians' competencies to manage care [14]. Similar emphasis resonated in the U.S. through the Henry Kaiser Family Foundation (KFF), America's Health insurance Plans (AHIP), and State Health Access Reform Evaluation (SHARE). KFF focused on demanding attention to necessary health policies [15], while AHIP's unified voice called for expanding access to high quality affordable coverage [16]. Also, SHARE [17] provided policy makers with evidence to allow access to affordable care to manage chronic illness.

\section{Health plans evolution}

The foundation of the health insurance system is grounded in employer-funded programs [11]. However, public funding of health insurance started in 1965 with the passing of the Medicare and Medicaid under Titles XVIII and XIX of the Social Security Act. Congress also assured maternal and child services under Title $\mathrm{V}$ as well as programs like Head Start [18]. The purpose was to address equitable access to health resources. With a reimbursement structure based on pay for services without control measures, costs escalated astronomically leading to $16 \%$ of gross domestic product (GDP) in 2005 [19].

Employer-based funding witnessed an explosive growth over the three decades following World War II (WWII) reaching a peak in 1980 which comprised $61 \%$ of coverage of non-elderly population in the U.S. [11,20]. It had major flaws involving inequitable share of costs, labor challenges, and inability to deliver cost-effective care. Changing coverage to managed care plans offered multiple products aimed at reducing costs through a network of providers with negotiated pay rates, limited drug choices and health benefits, and higher deductibles and premium rates [21].

\section{Health Plan Types}

Balancing the triad of cost, quality, and access to care remains a concern. Dominant health plan types attempting to balance this triad included consumer-directed health plans (CDHPs), preferred provider plans (PPOs), and comprehensive health plans (HMOs). The pairing of high deductible health plans (HDHPs) with health savings account (HSAs) proved to be flawed as only higher pay employees reaped the benefit [20,22].

CDHPs were also defined as HDHPs; the difference was inherent in the process of initiating health services [23]. PPOs, at $60 \%$ of market share, are the most common type of HDHPs [24] involving negotiated service network and contractual agreements for reimbursements. Over a two year period, premiums witnessed a rise of $81 \%$ for individuals and $59 \%$ for family coverage while the average deductible increased to $60 \%$. Ironically, the most common co-pay, physician's visit, was doubled
[24]. This raised concerns about health seeking behavior in response to these hikes.

HMOs carried the premise of control through network agreements and utilization of resources with pre-set guidelines and monitors for service delivery. Regardless of health plan, it was apparent that employees had no say in selection of benefit packages [20,25]. Instead, employees settled for their employer's choice of coverage.

\section{Cost shifting and affordability of care}

Access to care and affordability are two main concerns in the U.S. The working poor carry the brunt of affordability of the pricing of health services $[19,26]$. Whether the Massachusetts plan is a potential model for health reform remains to be seen [27]. In a meta-analysis of research studies, Hoffman and Paradise [11] emphasized the theme of affordability of care by low wage employees even when health coverage was available. The underinsured faced significant medical debts or delayed care.

Further, 19 million underinsured were in families that spent $20 \%$ of their income on health-related expenses, and more than $90 \%$ cited costs as a barrier to accessing care. Some employers do not offer coverage of dependents which further complicates affordability and subsequent health expenses [11,27]. Also, the "security of having health insurance is only temporary for many working adults" due to job loss. This resulted in one in three adults, or 52 million, reporting gaps in coverage over the two years prior to the study [12].

\section{Access to care}

When cost of care shifted to consumers, the intention was to control costs by giving consumers more "skin in the game" [28]. Nonetheless, findings suggested that individuals with HDHP had more inpatient days or hospital admissions related to skipping or delaying care due to cost $[28,29]$. Similarly, Chernew et al. [30] stated that compliance with medications declined when out-of pocket costs increased. The Institute of Medicine [1] also reported that working adults without health coverage were more likely to receive delayed care and had the tendency to be sicker or die sooner.

It is evident that the health delivery system in the U.S. is complex with negotiated agreements, restrictive health plans' network and utilization of resources, and consequent challenges in accessing care. This complexity posited loud cries for health reform. While some called for reform in payment of services [19,31], others focused on benefit structure and cost-sharing rules [3,11,32]. Regardless of needed reform, concerns about health coverage continued to dominate health reform debates.

Of interest in this study, was the continuity of health coverage which was synonymously used with continuity of care. The plight of the underinsured or "undercovered" working poor was highlighted by Weinstock [33] who stated that underinsurance forced a "waiting game" of delaying care, not filling prescriptions, skipping recommended tests or treatments, and not receiving specialty care. Thus, yielding care disruptions and negative health outcomes.

\section{Social Determinants of Health}

The literature also focused on personal attributes or social determinants of health and the relation to health-seeking behavior. Studies were inconclusive or inconsistent. Studies focusing on education level suggested that education level defined occupation or earnings which has more significance in influencing health $[9,34]$ examined literacy in a diabetes management program and found that health 
literacy can predict health status emphasizing the need for clinicians to adapt programs to literacy levels. Schillinger et al. [35], in a study of 408 participants, found patients with inadequate health literacy had poor glycemic control, $\mathrm{HgbA1C} \geq 9.5 \%$, and higher rate of retinopathy.

\section{Socioeconomic Status (SES)}

Researchers raised concerns about defining SES [36,37], and were consistent in viewing SES as multi-factorial and needing better measurement. Personal attributes like culture, faith, and perspectives on health were considered. The impact of beliefs and having a sense of community were addressed by Kickuchi's [38] reference to the value of "social goods" allowing equity in access to care and treatment.

Further, culture-sensitive care regulations such as culturally and linguistically appropriate care (CLAS), asserted the importance of culture and language in individualizing care delivery for more effective outcomes [39]. The standards called for matching care delivery to patients' cultural beliefs and preferred language. Culture care was emphasized in a study by Cohen and Martinez [40] who examined personal attributes and health plan selection. Their findings suggested that Hispanics and Non-Hispanic Blacks under age 65 were less likely to enroll in high deductible health plans (HDHP). This was attributed to ethnicity, cultural values of health as well as income and education level.

\section{Personal attributes}

With many strategies to influence health, a nagging concern about patients' engagement in treatment plans continues. Boyette et al. [7] in a meta-analysis of studies over 17 year period sought to understand determinants of health and clinicians' strategies in influencing participation in exercise programs. Personal attributes were defined as inclusive of age, gender, ethnicity or race, occupation, education level, and SES.

Similarly, the Institute of Medicine (IOM) Committee on the "Consequences of Underinsurance" (2009) reviewed 130 studies concerned with health insurance status and its effect on health outcomes for adults age $18-64$. The three dominant personal characteristics were: health status, race or ethnicity, and SES. Adults age 50-64 with lower income were more susceptible to declining health as evidenced by poor blood pressure control and higher risk of dying [41].

\section{Research Design}

In exploring the predictive value of underinsurance, continuity of health coverage, and health-seeking behavior on HR-QoL, I examined the strength and direction of the relationship between HR-QoL scores using SF12 $2^{2 \mathrm{ix}}$ and the independent variables while controlling for the covariates age, gender, race or ethnicity, income, and education level. I used multiple regression analysis to explore these relationships among the variables related to health.

\section{Research Questions}

I used the 0.05 level of significance to test the three research questions and hypotheses for the study and its analyses of Health Predictors for people ages 40 to 64 .

Research Question 1: To what extent does underinsurance and continuity of health coverage predict health-seeking behavior after controlling for age, gender, race or ethnicity, education level, and income?

Ho1: Underinsurance and continuity of health coverage will not significantly predict health-seeking behavior after controlling for age, gender, race or ethnicity, education level, and income.

H11: Underinsurance and continuity of health coverage will significantly predict health-seeking behavior after controlling for age, gender, race or ethnicity, education level, and income.

Research Question 2: To what extent does underinsurance, continuity of health coverage and health-seeking behavior predict health status after controlling for, age, gender, race or ethnicity, education, and income?

Ho2: Underinsurance, continuity of coverage, and health-seeking behavior will not significantly predict health status after controlling for age, gender, race or ethnicity, education, and income.

H12: Underinsurance, continuity of coverage, and health-seeking behavior will significantly predict health status after controlling for age, gender, race or ethnicity, education, and income.

Research Question 3: To what extent does health behavior by itself predict health status after controlling for age, gender, race or ethnicity, education, and income?

Ho3: There is no significant relationship between health-seeking behavior and health status after controlling for age, gender, race or ethnicity, education level, and income.

H13: There is a positive relationship between health-seeking behavior and better health status after controlling for age, gender, race or ethnicity, education level, and income.

\section{Dependent Variable (DV)}

\section{Health-related quality of life (HR-QoL)}

This variable reflects the participant's view of functionality, appearance, or comfort level that affected quality of life [32]. For the purpose of this study, HR-QoL was measured by a self-reported score using the QualityMetric instrument $S F 12 \mathrm{v} 2^{\mathrm{nw}}$, a tool that was recognized by the Agency for Health research and Quality (AHRQ) as valid and reliable [42]. Online scoring software for SF $12 \mathrm{v} 2^{\mathrm{max}}$ incorporates QualityMetric's MDE algorithm that provides summary scores for the physical and mental components of health.

\section{Independent Variables (IV)}

\section{Continuity of health coverage}

A Yes response to disruption in continued health coverage for three months or more over the last 24 months indicated a gap in coverage indicated continuity of coverage.

\section{Health-seeking behavior}

This variable was defined as care-seeking behavior described using four types of behavior: (a) avoiding care, (b) delaying care, (c) seeking care outside the health care system, or (d) contacting a physician or other clinician by phone or e-mail $[1,3]$. The health-seeking behavior tool had six questions. A Yes response had one (1) point while a No response had zero (0). The points were added to create a score that ranged from $0-6$. Then, a multiple linear regression was used to test a relation to underinsurance and health status.

\section{Underinsurance}

An individual with HDHP coverage that had to pay $\$ 1000$ or more a year before health coverage begins for health services was defined as underinsured. In this study, having HDHP = underinsured while a 
comprehensive health coverage $=$ Not underinsured .

\section{Covariates}

These were variables that have been consistently referred to in the health services literature as social determinants of health or personal characteristics that were related to health status [36]. These included:

Age: This variable was a self-reported number of years indicating age.

Gender: This was a dichotomous variable, male or female.

Race or ethnicity: This variable used four categorical variables that included: White (non-Hispanic), Black (non-Hispanic), Hispanic, and Other.

Education level: This variable was measured by the number of reported years as indicative of a respondent's level of education using 12 years as the mark for achieving high school level.

Income: In this study, income was self-reported as one of five ranges from less than $\$ 30,000$ to over $\$ 91,000$ a year. Responses were coded 1 through 5 based on the income category that was checked.

\section{Procedures}

Participants were selected from a hospital system with over 12000 employees in California. Systematic random sampling yielded 500 individuals that had either a high deductible health plan (HDHP) coverage, which was coded as 1 ; or HMO coverage coded as 0 . The sample pool depended on:

1. Power, "the likelihood of rejecting $H_{0}$ (i.e., avoiding a type II error)" where an $80 \%$ level is considered adequate

2. Effect size, "the degree to which $H_{0}$ is false

3. Significance level, the "probability of rejecting a true $H_{0}$ (making a type I error)"

Cohen asserted that effect size (ES), "the degree to which the phenomenon is present in the population", helps determine statistical power and necessary sample size to achieve a desired level of power with respect to a chosen ES that the researcher considers meaningful [43]. Since research question 2 had the most number of variables. Thus, I chose an ES about halfway between small and medium $\left(f^{2}=0.085\right)$. I also used $\mathrm{G}^{*}$ Power version 3.1.2 [44] to calculate the necessary sample size for power of $.95(n=207)$ and for power of $.80(n=133)$. Therefore, for a $50 \%$ response rate, I started with a sample of 500 . Surveys and reminder mailings yielded a total of $N=165$ respondents or $33 \%$ response rate instead.

\section{Inclusion and exclusion criteria}

Insured individuals under 40 and individuals over 64 years of age were not included in this study. Individuals that had publicly funded plans were excluded. Participants that were included met the age $40-64$ criteria and health plan type: HDHP or HMO. Surveys and informed consent forms were mailed explaining the study, voluntary participation, confidentiality and protection of their information, and availability of the researcher or IRB to answer any concern.

\section{The Study's Survey}

The survey had three sections: I. QualityMetric tool for measuring HR-QoL, SF $12 \mathrm{v} 2^{\mathrm{mix}}$, a valid and reliable instrument that was used for measuring patient-reported outcomes with more than $76,000,000$ surveys taken over 19,000 licenses for its use [45]. Section II was comprised of six health behavior questions that were tested in this study, and section III involved demographic questions.

\section{Data Analysis}

I examined the dependent variable, HR-QoL, through physical and mental components of health scores for 165 respondents using the QualityMetric instrument SF12v2 $2^{\mathrm{ix}}$. The independent variable, continuity of health coverage was dichotomous, measured by Yes response for having a health coverage gap of $\geq 3$ months or No response for having no interruption in coverage over the past two years. Relationships were tested with statistical significance set at $95 \%$ for greater confidence level. Further, a power analysis of 0.95 would allow a 95\% chance of finding an effect that allows rejection of the null hypothesis. I used SPSS software for data analysis and interpretation, and linear regression to examine the three research questions.

Protection of participants' rights was described in the informed consent that was mailed with the survey to potential participants. Voluntary participation and anonymity of participants' information were ascertained. Institutional review board (IRB) at the hospital system that provided the study's sampling pool ascertained the study met critical ethical guidelines for data collection, that is, having the researcher explain the purpose of the study, how the information will be used, and how participants' information will be protected.

\section{Findings}

Predictors of HR-QoL study was designed to explore relationships between health coverage type, continuity, health behavior, and health status as reflected by HR-QoL score. The goal was to fill a literature gap in empirically relating these variables and personal attributes to health status, and to validate the health behavior tool. Another goal was to examine the Health Belief Model (HBM) in a different way from its past use. I examined HBM as a decision making model influencing not only health behavior but also choice of health coverage. Last, my goal was to provide clinicians with effective strategies to individualize care; and to recommend a health policy for managing or paying for health services.

Using correlation methodology, I sought to answer three research questions regarding underinsurance, continuity of health coverage, health behavior, and HR-QoL. Multiple linear regression analyses were conducted to test the three hypotheses and assumptions of normality were applied. Discussion of findings covers descriptive statistics of the sample's demographics and analysis of the relationships among the study's main variables.

\section{Demographics}

The majority of respondents were female, accounting for $75.8 \%$ of the sample. Respondents were also mainly White ( $n=92$ or $55.8 \%)$, educated as demonstrated by 146 (88.4\%) out of total $N=165$ identifying their education level as either some college, college graduate, or some graduate level; and 142 as employed (86.1\%). The sample was consistent with the literature's respondents where the majority were White [31,34]; as well as having high education level when selecting HDHP $[28,40,46]$. Nearly two-thirds of participants, 98 (59.4\%) were married, and half of total $-84(50.9 \%)$ - earned more than $\$ 91,000$ annually. On average, the participants were 51 years of age and had two children. Table 1 shows frequency distribution and demographics.

\section{Health coverage}

Participants were categorized based on type of health coverage, deductible amount, and continuity of health coverage. Table 2 shows 


\begin{tabular}{|c|c|c|c|}
\hline Variable & Category & $\mathbf{n}$ & $\%$ \\
\hline \multirow[t]{2}{*}{ Gender } & Female & 125 & 75.8 \\
\hline & Male & 40 & 24.2 \\
\hline \multirow[t]{4}{*}{ Ethnicity } & White (non-Hispanic) & 92 & 55.8 \\
\hline & Other & 36 & 21.8 \\
\hline & Hispanic & 30 & 18.2 \\
\hline & Black (non-Hispanic) & 7 & 4.2 \\
\hline \multirow[t]{5}{*}{ Education } & Some college & 55 & 33.3 \\
\hline & College graduate (BA/BS) & 52 & 31.5 \\
\hline & Some graduate school or more & 39 & 23.6 \\
\hline & High school diploma/GED & 15 & 9.1 \\
\hline & Some high school & 4 & 2.4 \\
\hline \multirow{4}{*}{ Employment } & Currently employed & 142 & 86.1 \\
\hline & Other & 13 & 7.9 \\
\hline & Recently laid-off & 9 & 5.5 \\
\hline & Unemployed & 1 & .6 \\
\hline \multirow[t]{5}{*}{ Marital status } & Married & 98 & 59.4 \\
\hline & Divorced & 37 & 22.4 \\
\hline & Single/Never married & 22 & 13.3 \\
\hline & Separated & 4 & 2.4 \\
\hline & Widowed & 4 & 2.4 \\
\hline \multirow[t]{6}{*}{ Income level } & $\$ 91,000$ or more & 84 & 50.9 \\
\hline & $\$ 51,000$ to $\$ 70,000$ & 27 & 16.4 \\
\hline & $\$ 30,000$ to $\$ 50,000$ & 22 & 13.3 \\
\hline & $\$ 71,000$ to $\$ 90,000$ & 22 & 13.3 \\
\hline & Less than $\$ 30,000$ & 10 & 6.1 \\
\hline & Max. & M & SD \\
\hline Age & 64 & 51.75 & 7.18 \\
\hline Children & 8 & 1.54 & 1.39 \\
\hline
\end{tabular}

Note: $\mathrm{N}=165$

Table 1: Frequency Distribution and Central Tendency of Demographics for the Sample.

\begin{tabular}{|l|l|c|c|}
\hline Variable & Category & $\mathbf{n}$ & $\%$ \\
\hline Insurance type & PPO (Deductible $\geq \$ 1,000)$ & 50 & 30.3 \\
\hline & PPO (Deductible $\leq \$ 400)$ & 42 & 25.5 \\
\hline & HMO (Deductible $\leq \$ 400)$ & 40 & 24.2 \\
\hline & HMO (Deductible $\geq \$ 1,000)$ & 33 & 20.0 \\
\hline Continuity of coverage & No: no interruption in coverage & 144 & 87.8 \\
\hline & Yes: interruption in coverage & 20 & 12.2 \\
\hline
\end{tabular}

Note: $\mathrm{N}=165$ for insurance type and $\mathrm{N}=164$ for continuity of coverage.

Table 2: Frequency Distribution of Insurance Type and Continuity of Coverage.

the four categories used. The majority of participants, $144(87.8 \%)$, did not have a change or interruption in health coverage lasting over three months. There were no significant differences in HR-QoL scores, both physical components of health score (PCS) and mental component of health score (MCS), between the two groups: HMO and PPO. Therefore, the study failed to reject the null hypotheses.

\section{Health-seeking behavior}

The health behavior variable is used as DV in $\mathrm{H} 1$ and IV in $\mathrm{H} 2$ and H3. To construct this variable, the sum of six questions' responses was used. The frequency distribution is presented in Table 3. Cronbach's alpha was calculated to measure the reliability of the health behavior tool. The alpha value was .63. This finding is further discussed within the limitations section.

Neither hypothesis 1 or 2 had underinsurance as a sole predictor of health behavior or HR-QoL. Findings did not allow me to reject the Null Hypotheses 1 and 2. However, the predictability of healthseeking behavior with underinsurance and continuity of coverage was significant. The inclusion of continuity of coverage significantly increased the amount of variance in health seeking behavior, from $7.2 \%$ with underinsurance alone to $12.6 \%$, with the addition of continuity of coverage. This finding was also reflected by Pearson Correlation in Table 4 indicating statistically significant relationship between continuity of health coverage and health seeking behavior, and in Table 5 where uninterrupted health coverage increased health seeking behavior by

\begin{tabular}{|c|c|c|c|c|}
\hline & \multicolumn{2}{|c|}{ No } & \multicolumn{2}{|c|}{ Yes } \\
\hline Question & $\mathrm{n}$ & $\%$ & $\mathrm{n}$ & $\%$ \\
\hline 1. Delayed seeing a physician or primary source of care. & 119 & 72.1 & 46 & 27.9 \\
\hline $\begin{array}{l}\text { 2. Did not have a regular primary source of care } \\
\text { (Physician, Nurse Practitioner, or Clinic). }\end{array}$ & 122 & 73.9 & 43 & 26.1 \\
\hline $\begin{array}{l}\text { 3. Did not have a visit to the doctor (Call or e-mail } \\
\text { instead). }\end{array}$ & 128 & 77.6 & 37 & 22.4 \\
\hline 4. Did not have a recommended test. & 119 & 72.1 & 46 & 27.9 \\
\hline 5. Could not pay medical bills. & 146 & 88.5 & 19 & 11.3 \\
\hline 6. Did not fill prescription medications. & 148 & 89.7 & 17 & 10.3 \\
\hline
\end{tabular}

$N=165$.

Table 3: Frequency Distribution of the Six Questions Comprising the Health Behavior Score

\begin{tabular}{|l|c|c|c|c|} 
& \multicolumn{4}{|c}{ Pearson Correlations on Study Variables } \\
\hline Study Variable & 2 & 3 & 4 & 5 \\
\hline 1. Underinsurance & .15 & -.01 & .06 & .01 \\
\hline 2. Continuity of Health Coverage & -- & $.26^{\star *}$ & .05 & .12 \\
\hline 3. Health-seeking behavior & -- & -- & .06 & .11 \\
\hline 4. Physical health status (HR-QoL) & -- & -- & -- & .13 \\
\hline 5. Mental health status (HR-QoL) & -- & -- & -- & -- \\
\hline
\end{tabular}

Note. ${ }^{*} p<.05$

Table 4: Pearson Correlations Used to Analyze the Relationships between the Study Variables.

\begin{tabular}{|l|c|c|c|c|}
\hline & \multicolumn{4}{|c|}{ Health-Seeking Behavior } \\
\hline Variable & Model 1 B & Model 2 B & Model 3 B & $95 \% \mathrm{Cl}$ \\
\hline Constant & 1.30 & 1.22 & 1.26 & {$[-.62,3.15]$} \\
\hline Age & -.01 & -.01 & -.01 & {$[-.04, .02]$} \\
\hline Gender & -.21 & -.21 & -.20 & {$[-.71, .30]$} \\
\hline $\begin{array}{l}\text { Ethnicity } \\
\text { (White vs. Other) }\end{array}$ & .10 & .11 & .13 & {$[-.10, .36]$} \\
\hline $\begin{array}{l}\text { Some college or less vs. } \\
\text { College graduate BA/BS }\end{array}$ & .06 & .04 & .06 & {$[-.49, .60]$} \\
\hline $\begin{array}{l}\text { Some college or less vs. Some } \\
\text { graduate school }\end{array}$ & .56 & .57 & .55 & {$[-.05,1.15]$} \\
\hline $\begin{array}{l}\$ 91,000 \text { or more vs. Less than } \\
\$ 50,000\end{array}$ & $.82^{\star}$ & $.84^{*}$ & .62 & {$[-.03,1.27]$} \\
\hline $\begin{array}{l}\$ 91,000 \text { or more vs. } \$ 51,000 \\
\text { to } \$ 70,000\end{array}$ & .04 & .06 & -.02 & {$[-.67, .64]$} \\
\hline $\begin{array}{l}\$ 91,000 \text { or more vs. } \$ 71,000 \\
\text { to } \$ 90,000\end{array}$ & .38 & .40 & .30 & {$[-.39,1.00]$} \\
\hline Insurance (HMO vs. PPO) & -- & .12 & -.01 & {$[-.47, .46]$} \\
\hline Continuity of coverage & -- & -- & $1.07^{\star *}$ & {$[.38,1.75]$} \\
\hline $\mathrm{R}^{2}$ & .070 & .072 & & .126 \\
\hline $\mathrm{F}$ & 1.46 & 1.33 & \multicolumn{2}{|c|}{$2.20^{*}$} \\
\hline$\Delta \mathrm{R}^{2}$ & & .002 & \multicolumn{2}{|c|}{.054} \\
\hline$\Delta \mathrm{F}$ & & .27 & \multicolumn{2}{|c|}{$9.39^{* *}$} \\
\hline
\end{tabular}

Note: $\mathrm{Cl}=$ Confidence Interval.

${ }^{*} \mathrm{p}<.05^{* *} \mathrm{p}<.01$

Table 5: Multiple Linear Regression Using Underinsurance and Continuity of Coverage to Predict Health-Seeking Behavior. 


\subsection{However, I was not able to reject the Null Hypothesis 1.}

Although Health behavior did not significantly contribute to predicting health status for either group (underinsured or not underinsured), data analysis showed an increase in health behavior corresponded to an increase in HR-QoL scores, PCS and MCS, thus indicating a positive relationship. I was not able to reject hypothesis 3 but there was a positive correlation between health behavior and health status, which was the purpose of choosing the effect size I used. However the study's limitation did not allow generalizability of findings.

Inferences about the Health Belief Models (HBM) for decision making were not made when examining findings due sampling and health behavior tool's limitations. HBM's four constructs relating to perceived susceptibility to illness, seriousness of disease or illness, benefits of taking actions or barriers to action, and cues to action as in seeking care could not be asserted because the perspective of the working poor was not captured as a result of sampling limitation. However, where there was continuity of health coverage with the majority of participants in the high income bracket, decisions for health seeking behavior clearly increased. Similarly for health coverage type, inferences about HBM and decisions to select specific coverage type were not made due to a sample dominated by one ethnic group that was also affluent in terms of income and education level as discussed in the study's limitations.

\section{Limitations}

There were two main limitations in this study. One area involved the tool I used to measure health behavior, and the second involved sampling. With unrepresentative sample, the study's generalizability was limited.

\section{Health behavior tool}

Section II of the study's survey had six questions tool for measuring health behavior. Despite its use in multiple survey-based studies, testing the reliability of this tool presented a literature gap. Cronabch's alpha test for this study was .63 which is considered questionable thus, posing a limitation of this study. Cronbach's alpha coefficient is a measure of internal consistency or reliability of an instrument that provides "beginning evidence of construct validity" for developing the tool as a scale [47]. The literature I reviewed did not yield a clear value for an acceptable Cronbach's alpha. Neuendorf [48] stated that an acceptable value was dropped down to .60 or .70 from past acceptable range of $.80-.90$

Furthermore, the acceptable value for a Cronbach's alpha varied with the number questions in a tool when considering the interrelatedness or homogeneity of the questions [49,50]. Gliem and Gliem [51] citing George and Mallery, stated an often referenced rule-ofthumb of Cronbach's alpha value being: $>.9$ is excellent, $>.8$ is good, $>.7$ is acceptable, and $>.6$ is questionable. In this study, the health behavior tool was .63 which makes it less than acceptable.

\section{Sampling limitations}

With the majority of participants being White (55.8\%), female (75.8\%), with high education level (86.1\%), and income level (50.9\%); it was clearly a biased sample. Another limitation involved the wide age range of 40-64. With an average participant's age of 51, capturing the perspectives of individuals in the upper age range was limited. Further, perspectives of Black, Hispanic, or other ethnicity as well as perspectives of those in lower income and education levels could not be captured.

The literature had consistency in survey respondents being White, with higher income and education levels. Thus, the views of individuals that do not respond to surveys (non-responders) was yet again, not captured. This leads to biased interpretation of findings in survey-based studies $[52,53]$.

\section{Conclusion}

Affordability of care remains a major concern for many Americans. Over the years, cost-shifting to individuals occurred. Also, accountability for seeking and adhering to treatment or preventive-care measures shifted as well. Recent health initiatives emphasized patients' engagement in care and set expectations for providers to follow [54]. These initiatives held every entity accountable for a partnership between payers, providers of care, and patients in an effort to achieve balance between quality, cost, and access. In this study, I sought to explain this patient engagement and selection of health coverage through an understanding of health-seeking behavior. Continued health coverage surfaced as a dominant theme for increasing health-seeking behavior.

\section{Social change implications}

I did not find significant relationships between the predictor variables and HR-QoL in this study. Limitations of sampling and health behavior tool contributed to lack of confidence in rejecting the null hypotheses. However, I was able to find that continued health coverage and health behavior were positively related. The Pearson Alpha Correlation indicated a statistically significant relationship between continuity of health coverage and health seeking behavior, $r(162)=.26, p<.01$. This finding is useful in clinical practices focusing on patient engagement for seeking health services or following directions of care regimens. A better understanding of health behavior by clinicians encourages patient engagement and potentially yields better outcomes. Recent initiatives for collaborative care by the Center for Medicare \& Medicaid Services (CMS) emphasized the value of patients' engagement in care [55]. Similarly, Tower-Watson, the National Business Group on Health (TW/NBGH) found that lack of patients' engagement was the biggest barrier to changing health behavior [56]. This has implications for supporting strategies focusing on patients' engagement in influencing health status. Understanding conditions for enhancing health-seeking behavior, as this study found with continued health coverage, has that potential. During patients' assessments, clinicians should query their patients about disruptions in their health coverage and the duration of interrupted coverage. This can guide clinicians' orders or referrals for health screenings and therapeutic treatment measures to make up for the gap in continued care and yield earlier diagnostics or interventions.

Although the study's sample was not representative of the population, respondents had $12.6 \%$ variance in health seeking behavior with uninterrupted health coverage. This emphasis on the value of continued health coverage helps the underinsured continue to seek timely diagnostic and therapeutic. Perhaps an implication for positive social change is warranting a health policy that addresses affordability of care for low-income earners. This recommendation is the study's contribution to social change and to supporting social initiatives that focus on patients' engagement for healthy living.

\section{Recommendations for practice}

I had three detected variances that can potentially allow desired outcomes of care delivery. These involve the personal attributes of education and income, health seeking behavior, and continuity of health coverage. In this study, I found high scores of physical and mental health where $88.4 \%$ of respondents were educated and $50.9 \%$ earned more than $\$ 91000$ annually. It is apparent that clinicians should focus 
on outreaching the less educated and low wage earners for managing illness and providing health and wellness services. Perhaps initiatives like value-based purchasing or value-based care should include care outcomes involving these groups of patients to get a clear picture of clinicians' value and contribution to health outcomes.

Additionally, I was able to find a $5.4 \%$ increase in variance when continuity of health coverage was added to the analysis suggesting an effect size may exist. Most likely, it is a good clinical judgment to focus on continuity of care as contributory to better health outcomes, and include continued health coverage query in assessments and planned interventions. Last, health-seeking behavior increased when health coverage continued without interruptions. This finding emphasizes the value of maintaining health coverage at the individual, regulatory, and policy-making levels. As national collaborative initiatives involve patients' engagement in care, policy-making should account for providing continued health coverage prior to holding patients accountable for their care. It is evident that individuals decide to drop health coverage if they are unable to pay for it. While terminated or laid-off employees may be entitled to continue insurance coverage under the Consolidated Omnibus Budget Reconciliation Act (COBRA) regulations, they are likely to drop their health coverage due to lack of steady income and employers' subsidies of premiums that were granted during the period of active employment [27,57]. Based on this study's findings, a proposal to amend COBRA regulations is warranted to force either continued employer's subsidy of premiumsor to offer subsidy of premiums with unemployment benefits. Consequently, a status of continued health coverage that allows individuals access to care and wellbeing can be realized. In order to realize national initiatives for patient engagement, a policy proposal involves either an amendment to COBRA or drafting a new health policy for maintaining employee's share of premiums throughout that 18 months period of COBRA. This allows individuals to maintain a status of health and wellbeing until other options for health coverage are feasible.

Key recommendations from this study are: a repeat analysis of continuity of health coverage and HR-QoL, and testing the health behavior tool. There is a need to focus on individuals transitioning through careers and an aging process. Further, allowing better access to primary care may yield better health outcomes and mitigate the effect of chronic conditions on health and health care spending. Having a healthy nation is a social responsibility, and health policies must address the issue of continued health coverage and premiums' subsidy so that equal access to care and health-related quality of life are available to all.

\section{References}

1. Institute Of Medicine (IOM) Report (2003) Care without coverage: Too little, too late.

2. National Committee on Quality Assurance [NCQA] (2010) California Pay for Performance (P4P).

3. Reed M, Fung V, Price M, Brand R, Benedetti N, et al. (2009) High-deductible health insurance plans: efforts to sharpen a blunt instrument. Health Aff (Millwood) 28: 1145-1154

4. Schoen C, DesRoches C (2000) Uninsured and unstably insured: the importance of continuous insurance coverage. Health Serv Res 35: 187-206.

5. Busch SH, Barry CL, Vegso SJ, Sindelar JL, Cullen MR (2006) Effects of a costsharing exemption on use of preventive services at one large employer. Health Aff (Millwood) 25: 1529-1536.

6. Shea DG, Short PF, Powell MP (2001) Betwixt and between: targeting coverage reforms to those approaching Medicare. Health Aff (Millwood) 20: 219-230.

7. Boyette LW, Lloyd A, Boyette JE, Watkins E, Furbush L, et al. (2002) Personal characteristics that influence exercise behavior of older adults. J Rehabil Res Dev 39: $95-103$

8. Montori VM, Bryant SC, O'Connor AM, Jorgensen NW, Walsh EE, et al. (2003)
Decisional attributes of patients with diabetes: the aspirin choice. Diabetes Care 26: 2804-2809.

9. Rothman RL, DeWalt DA, Malone R, Bryant B, Shintani A, et al. (2004) Influence of patient literacy on the effectiveness of a primary care-based diabetes disease management program. JAMA 292: 1711-1716.

10. Schrop SL, Pendleton BF, McCord G, Gil KM, Stockton L, et al. (2006) The medically underserved: who is likely to exercise and why? J Health Care Poor Underserved 17: 276-289.

11. Hoffman C, Paradise $\mathrm{J}$ (2008) Health insurance and access to health care in the United States. Ann N Y Acad Sci 1136: 149-160.

12. Hoffman C, Schoen C, Rowland D, Davis K (2001) Gaps in health coverage among working-age Americans and the consequences. J Health Care Poor Underserved 12: 272-289.

13. Baker DW, Sudano JJ, Albert JM, Borawski EA, Dor A (2001) Lack of health insurance and decline in overall health in late middle age. N Engl J Med 345 1106-1112.

14. World Health Organization (WHO) (2005) Preparing a health care workforce for the $21^{\text {st }}$ century: The challenge of chronic conditions. WHO Publications, Geneva, Switzerland.

15. The Henry J Kaiser Family Foundation (KFF) (2010) About the Kaiser family foundation.

16. America's Health Insurance Plans (AHIP) (2010) About AHIP.

17. The State Health Access Reform Evaluation (SHARE) (2010) State of reform contacting SHARE.

18. Richmond JB, Fein R (1995) The health care mess. A bit of history. JAMA 273 69-71.

19. Banthin JS, Bernard DM (2006) Changes in financial burdens for health care: national estimates for the population younger than 65 years, 1996 to 2003 JAMA 296: 2712-2719.

20. Enthoven AC, Fuchs VR (2006) Employment-based health insurance: past, present, and future. Health Aff (Millwood) 25: 1538-1547.

21. Sered SL, Fernandopulle R (2005) Uninsured in America: Life and death in the land of opportunity. University of California Press, Berkley, CA.

22. Wilensky GR (2006) Consumer-driven health plans: early evidence and potential impact on hospitals. Health Aff (Millwood) 25: 174-185.

23. Newhouse JP (2004) Consumer-directed health plans and the RAND Health Insurance Experiment. Health Aff (Millwood) 23: 107-113.

24. Claxton G, Gabel J, Gil I, Pickreign J, Whitmore H, et al. (2006) Health benefits in 2006: premium increases moderate, enrollment in consumer-directed health plans remains modest. Health Aff (Millwood) 25: w476-w485.

25. Blumenthal D (2006) Employer-sponsored health insurance in the United States--origins and implications. N Engl J Med 355: 82-88.

26. Berk ML, Gaylin DS, Schur CL (2006) Exploring the public's views on the health care system: a national survey on the issues and options. Health Aff (Millwood) 25: w596-w606

27. Jost TS (2008) Access to health care. Is self-help an answer? J Leg Med 29: 23-40

28. Bloche MG (2006) Consumer-directed health care. N Engl J Med 355: 1756 1759.

29. Dixon A, Greene J, Hibbard J (2008) Do consumer-directed health plans drive change in enrollees' health care behavior? Health Aff (Millwood) 27: 1120-1131.

30. Chernew ME, Shah MR, Wegh A, Rosenberg SN, Juster IA, et al. (2008) Impact of decreasing copayments on medication adherence within a disease management environment. Health Aff (Millwood) 27: 103-112.

31. Schoen C, Doty MM, Collins SR, Holmgren AL (2005) Insured but not protected: how many adults are underinsured? Health Aff (Millwood) Suppl Web Exclusives: W5-289-W5-302.

32. Ginsburg M, Goold SD, Danis M (2006) (De)constructing 'basic' benefits: citizens define the limits of coverage. Health Aff (Millwood) 25: 1648-1655.

33. Weinstock M (2007) The Undercovered Class. Hospitals \& Health Networks.

34. Miech RA, Hauser RM (2001) Socioeconomic status and health at midlife. A comparison of educational attainment with occupation-based indicators. Ann Epidemiol 11: 75-84 
35. Schillinger D, Grumbach K, Piette J, Wang F, Osmond D, et al. (2002) Association of health literacy with diabetes outcomes. JAMA 288: 475-482.

36. Braveman PA, Cubbin C, Egerter S, Chideya S, Marchi KS, et al. (2005) Socioeconomic status in health research: one size does not fit all. JAMA 294: 2879-2888.

37. Shavers VL (2007) Measurement of socioeconomic status in health disparities research. J Natl Med Assoc 99: 1013-1023.

38. Kikuchi JF (2005) Cultural theories of nursing responsive to human needs and values. J Nurs Scholarsh 37: 302-307.

39. US Department of Health and Human Services (DHHS), Office of Minority Health (2010) National standards for culturally and linguistically appropriate services in health care.

40. Cohen RA, Martinez ME (2009) Consumer-directed health care for persons under 65 years of age with private health insurance: United States, 2007. NCHS Data Brief: 1-8.

41. Committee on Quality of Health Care in America, Institute of Medicine (2001) Crossing the quality chasm: A new health system for the 21 st century. National Academies Press, Washington, DC.

42. Quality Metric (2010) Using the SF-36 and SF-12 health surveys to predict medical expenses.

43. Parker RI, Hagan-Burke S (2007) Useful effect size interpretations for single case research. Behav Ther 38: 95-105.

44. Faul F (2009) G*Power Version 3.1.2.Concept and design. Copyright ${ }^{\oplus} 2003$ 2009.

45. QualityMetric (2010) Generic health surveys.

46. Buntin MB, Damberg C, Haviland A, Kapur K, Lurie N, et al. (2006) Consumerdirected health care: early evidence about effects on cost and quality. Health Aff (Millwood) 25: w516-530.
47. Munro BH (2005) Statistical methods for health care research (5 $5^{\text {th }}$ ed.). Philadelphia, PA: Lippincott Williams \& Wilkins.

48. Neuendorf K (2003) Internal consistency reliability: Can Cronbach's alpha be too high?

49. Grau E (2007) Using factor analysis and Cronbach'salpha to ascertain relationships between questions of a dietary behavior questionnaire. Proceedings of the Survey Research Methods Section, ASA.

50. Tavakol M, Dennick R (2011) Making sense of Cronbach's alpha. Int J Med Educ 2: 53-55.

51. Gliem JA, Gliem RR (2003) Calculating, Interpreting, and Reporting Cronbach'salpha reliability coefficient for Likert-type scales.

52. Porter SR, Whitcomb ME (2005) Non-Response in student surveys. The role of demographics, engagement and personality. Research in Higher Education 46: 127-152.

53. Woosley SA (2005) Survey response and its relation to educational outcomes among first-year college students. Journal of College Student Retention: Research, Theory, and Practice 6: 413-423.

54. HealthCare.gov (2012) Partnership for patients: Better care, lower costs

55. Centers for Medicare \& Medicaid Services (2012) Comprehensive primary care initiative.

56. Tower-Watson/National Business Group on Health (TW/NBGH) (2012) Performance in an era of uncertainty: Employer survey on purchasing value in health care.

57. Kapur K, Marquis MS (2003) Health insurance for workers who lose jobs: implications for various subsidy schemes. Health Aff (Millwood) 22: 203-213. 